\title{
Managing Risk in the Real World
}

We have put forward a series of arguments culminating in the idea that patient safety should be viewed as the management of risk over time. We have suggested that healthcare could draw on a much wider repertoire of strategies and interventions to manage risk and enhance safety. This has been a book of ideas and argument but we hope that these are both rooted in practice and have practical application. In this chapter we first consider some of the more immediate implications as we see them and then consider the form a longer term exploration and development might take.

\section{Implications for Patients, Carers and Families}

The engagement of patients in patient safety has been a slow and difficult process. Much of the initial effort has gone into engaging patients alongside staff in reporting and acting on safety issues. This has been a valuable exercise but there is always (rightly) going to be a limit on what it is reasonable or feasible for patients to take on in hospital. We should now turn our attention to the home and community which will pose very different safety challenges. For instance, nosocomial infections are common in hospitals but we have developed effective ways of countering them which rely on close monitoring and a rapid clinical and organisational response. In the home, the risk of nosocomial infections may be less but other risks arise from the open environment, frequent visitors and varying standards of hygiene. Safety is a moving balance between accepted risks and available solutions; you can improve safety either by changing the exposure to risk or improving solutions.

In the home and community patients are in charge of care, and therefore responsible for safety, capable of making errors and being influenced by the many factors that affect safety. This is more than engagement, shared decision making or partnership. Patients and families are taking on roles and responsibilities that are in other settings restricted to professionals. This raises a host of issues for the management of risk and indeed for the delivery of services generally. 
We know that patients and families take safety very seriously and are ingenious in managing many potentially dangerous scenarios. We have given examples in the book and no doubt many more could be collected and studied to reveal novel strategies and interventions which could be shared, adapted and potentially used more widely. Our five strategies can be used to pose some immediate questions about the risks managed by patients and families. What training should be given? If a professional needs training to, for instance, change a dressing while maintaining sterile conditions then surely patients and carers need training too. To what extent can standards of hygiene be relaxed simply because a sick person has moved from hospital to home? We may need to consider setting standards and controlling the environment in which care can be delivered. What kind of support do patients and families need if they are to monitor safety and act appropriately on signs of deterioration? The example of home haemodialysis given earlier shows that advanced units are now including a suite of safety strategies in their training for patients and families. This could potentially be replicated, in varying degrees of intensity, for other forms of care outside hospital.

\section{Implications for Frontline Clinicians and Managers}

In healthcare the word frontline is generally taken to mean clinical staff in direct contact with patients and whose actions and decisions have immediate effects. Managers do not deliver treatment and so are not frontline in that sense. They are frontline however in the sense that the actions of clinical managers have a very powerful influence on safety. A bed manager in a large hospital for instance is constantly juggling patients and beds, assessing the latest request for an urgent bed, trying to place patients in wards that are at least reasonably appropriate and preventing very sick patients being in wards where the staff are not familiar with their needs. 'Being in the wrong place' is high risk if you are very sick. Clinical managers have a huge influence on safety but we know little about the strategies they use.

Both clinicians and managers can do a great deal to improve the standards and value of incident analysis. In the United Kingdom at least what was once an exercise in learning, reflection and improvement has, in some settings, sadly deteriorated into a largely bureaucratic exercise producing numerous recommendations that can never be implemented. There is an urgent need to return to the original purpose of incident analysis, focus on the comprehensive investigation of a much smaller number of events and consider the findings in the context of an overall safety and quality improvement programme. This can all be achieved with methods we already have. We also however need to explore the analysis of episodes of care with the attendant attention to contributory factors at different points, adaptation and recovery from problems, and much greater attention to the accounts of patients and families.

We believe that patients and families should select a proportion of the analyses and be encouraged to contribute as much as they can to analyses; their perspective is obviously particularly critical outside hospital. Their perspective will help us understand the longer term safety problems and to develop new techniques and 
innovations. This perspective might seem utopian and to require huge resources; it would certainly require some careful organisation and the use of technology to bring in some participants. As before though, quality is more important than quantity. A relatively small number of thorough investigations can produce a huge amount of useful information about the vulnerabilities, defences and resilience of the healthcare system.

Frontline teams, with management support, can initiate a much wider and more strategic programme of risk management than is currently the case. We could envisage the development of a decision tree in which different strategies and interventions could be considered sequentially, both separately and in combination, as candidates to enhance safety in any particular setting and in response to identified problems. Improving standards of practice is the most common approach to safety on the frontline and, if achievable, is an obvious and necessary first step. Next there are multiple ways of improving the wider system, though many are not in the control of frontline teams. A critical task is to identify points in the system where inefficient processes and poor reliability are forcing time wasting and potentially dangerous workarounds; the adaptations are of course necessary at the time but wasteful in that they are simply a compensation for other deficiencies rather than a necessary response to problems or crisis. Coping in the short term is admirable and may be in the best interests of that particular patient but the longer term this attitude is detrimental to safety in that it simply prolongs the underlying problems and removes any incentive for change. Risk controls, achieved with professional and management consensus, protect both patients and staff and could bring order and calm to currently chaotic systems. In emergencies of course risk controls can and should be over-ridden.

Monitoring, adaptation and response can be misused but is nevertheless an absolutely critical safety strategy at every level of the system. A great deal has been achieved in team training in anaesthesia, surgery, emergency medicine and other clinical contexts. The skills of monitoring, cross checking and other features of human factors team training are widely taught and such programmes have been shown to improve safety and clinical outcomes. We need to devote much more energy to understanding how people at every level of the system adapt and respond to safety critical issues and develop methods of preparation and training in these skills.

One important direction of travel would be a parallel exploration of how these and other strategies are used by managers, particularly those directly involved in clinical services. Managers constantly adapt and firefight; how much is necessary and how much unnecessary and due to poor systems? Which strategies and interventions are currently used day-to-day and at times of crisis and which would be optimal? We in no way wish to denigrate the skill and dedication of managers who go to extraordinary lengths to maintain safety. Rather, we want to move away from ad hoc improvisation towards explicit and planned interventions, preparation and training in the use of a portfolio of strategies and interventions. A huge amount could be learned from studying the ways managers adapt and cope and by refining this into a more strategic approach. A customised safety training programme for 
managers, or perhaps pairs of managers and clinicians, would be high on our wish list for the future of safety.

\section{Implications for Executives and Boards}

In the United Kingdom and some other countries boards governing healthcare institutions include people from other sectors who bring very different expertise and perspectives. To an engineer, for instance, it can be very difficult to appreciate that what is tolerated in healthcare is very different from what is tolerated in engineering. Incidents brought to the attention of boards are often understood as horrifying and unusual departures from best practice, rather than as the inevitable by-product of the multiple vulnerabilities of an overstretched system. The most critical realisation at board level is the recognition of the extent of poor reliability, difficult working conditions and the corresponding necessity for ad hoc improvisation and cutting corners that is frequently necessary and often actively encouraged. Even clinical members of boards, who know this from daily experience, may struggle to make this explicit. This is a necessary background understanding to any effective action on safety and the inevitable compromises and trade-offs necessary in the delicate and fluctuating balance between finance, safety, quality and patient experience.

An important observation in the implementation of the recently developed framework for the measurement and monitoring of safety has been that the core ideas appear to resonate in different settings and at different levels of the healthcare system. This is valuable in that an organisation could potentially cohere around a core set of safety questions which are meaningful to staff at all levels. We do not know how our framework of strategies and interventions will be received and to what extent they will be applicable at different levels of the system. We are conscious that the language and practice of safety improvement is more akin to frontline practice, while the language of control, assurance and mitigation are more familiar to those at executive, regulation and policy level. It would be enormously valuable if the safety community could find a language and practice that spanned all levels and contexts, and which resonated with patients, frontline staff, executives and regulators alike. We believe that it is achievable and could provide a much needed clarification and integration of safety initiatives.

Boards too can employ a much wider range of strategies and interventions. A strategic combination of approaches and interventions is necessary to achieve optimal safety in the face of financial restrictions and constraints. An expansion of safety strategies may allow them to employ approaches such as risk control which are more familiar and akin to those employed in the management and oversight of finance. Boards often associate improving safety with spending more money, but a judicious combination of strategies and interventions may allow safety interventions to at least be cost neutral overall. One might imagine for instance that controls, restrictions and improved reliability would reduce costs and permit the development of a programme for managers aimed at optimising the simultaneous management of safety, cost, quality and patient experience. This also may sound optimistic but we 
believe is possible given a sufficiently wide and well thought out safety programme.

At this level of an organisation the integration of strategies and programmes and the explicit trade-off between objectives is a critical skill. An organisational or regional change strategy is generally a combination of individual sub-programmes developed and led by different directors. The individual programmes almost inevitably conflict with each other. For example the ideal plan for reducing the debt at a satisfactory pace is generally detrimental to investments in staff and new technology and ultimately quality and safety.

The development of the final strategy will rest ultimately with the Chief Executive, the board and a small group of senior leaders. They must arbitrate between the individual directors and programmes, create and maintain an overarching vision which encompasses all the objectives of the organisation. There are good and bad ways of achieving these compromises; each director must be willing to adapt his or her particular programme and integrate with other organisational objectives and plans. The Chief Executive and other senior leaders need to be skilled in arbitrating and negotiating with all concerned to achieve a plan which achieves the objectives of the organisation without unduly compromising frontline quality and safety.

Scenarios of this kind are common currency in business school executive programmes but they very seldom include safety issues, at least for healthcare. Developing scenarios in which safety is managed in a realistic and clear sighted way in the face of financial pressures would be a major step forward in the management of risk. A particularly critical issue is the recognition of the early signs of organisational failure, both for those running organisations and for those attempting to monitor them externally such as regulatory agencies and government. Executive courses aimed specifically at the development of strategies which simultaneously address safety, finance and other organisational objectives are being developed and trialled in the oil and gas industries but have not yet been initiated in healthcare.

\section{Implications for Regulatory Agencies and Government}

Regulatory agencies face some major new challenges. Until now most regulation has focused on individual healthcare professionals or specific organisations and institutions. Regulation in its various forms now needs to extend to encompass new organisational forms and the complex series of transitions and interfaces along the patient journey. The accreditation of new types of organisation is already in progress in many countries but still requires further development; it is often not clear, for instance, what jurisdiction regulators have over patients living relatively independently in residential care. Traditional approaches to certification, inspection, and the corresponding evaluation criteria may have to be adapted considerably. To move from accreditation of structures and institutions to accrediting patient journeys across primary, secondary and home care is a huge challenge. 
A second major challenge is to find a way of regulating a very rapidly evolving system. Regulators in most other industries are blessed with a relatively static environment in which standards can be set and maintained over years or even decades; there is innovation of course but it does not usually lead to a change in core standards, simply a better way of meeting them. In aviation or the nuclear industry major changes may take 10 years from initial proposal to eventual implementation allowing ample time for the development of professional consensus, formal trials and the gradual absorption into the regulatory framework.

In contrast, the rapid pace of innovation in investigations and treatments in healthcare means that the regulator inevitably lags behind innovation. The fast pace of innovation makes developing new standards very challenging; standards can be developed quickly and adapted to a rapidly changing environment but only with a consequent reduction in rigour and testing, since formal evaluation cannot possibly be achieved within the time available. The present system cannot cope with the pace of innovation but it is far from clear how to develop new and more responsive modes of regulation.

Politicians and others at very senior level are under pressure to maintain the fiction that every citizen can have optimal healthcare. In private at least, it is absolutely critical that government and regulators recognise the vulnerabilities of the system and the gap between what is intended and what is actually delivered. The idea of absolute standards is naïve and potentially dangerous especially for struggling organisations. Innovation and the implementation of new and improved standards, all desirable, place huge pressures on both individual organisations and the wider system and create new safety issues. Many regulatory agencies understand this very well but may nevertheless struggle to find an effective response to the issue. The problem of regulation is often conceived as the problem of finding good ways to detect this gap and identify poorly performing organisations. It is essentially a 'best practice' view of safety. However, the deeper problem of regulation is not so much identifying the departure from standards but about how to manage that gap intelligently and humanely. The problem in our terms is one of monitoring, adaptation and response and to develop approaches that are strategic rather than improvised.

Regulatory agencies have developed very comprehensive approaches to inspection and have devoted most of the energies to monitoring compliance with standards. Much less attention has been given to the critical issue of how to respond when standards are not achieved. In many cases the response seems little more than admonition, threats and re-inspection. A basic risk control strategy would mean closing or limiting facilities when an inspection reveals fundamental problems but this threat is usually met with strong local resistance. The healthcare system either needs to overcome these obstacles or take stock and accept that no facilities cannot and develop a more sophisticated response to lapses in standards. We need, just as at other levels of the system, to consider how organisations and regulators might work together in a process of adaptation and ongoing monitoring of the gap between the ideal and the real. Delay in bringing the organisation to the point of compliance with standards, which can take months or even years, can be dangerous but there is seldom any explicit discussion of how to manage safety in the interim. The art of 
negotiation of realistic timescales for change and compliance needs exploration, research and development.

\section{Future Directions for Research and Practice}

This short book and these proposals are a first step. We believe that there are immediate implications but recognise that if the ideas have merit then they need to be debated, developed further and tested in practice by a community of people. The table in Chap. 11 provides, as we expressed it, an incomplete taxonomy. We know that much more work is need to map the full set of strategies and interventions, assess the value of the overall framework, the nature and purpose of the various interventions and their effectiveness in practice. Our experience so far from the small group of people who generously found time to read an earlier draft is that they recognised the need for a broader view of safety, for a breadth of strategic approach and particularly to the need to customise approaches to safety to different settings and along the patient journey.

The next step is broadly ethnographic. We need to observe, identify and collate safety relevant strategies and interventions at all levels of healthcare organisations and the wider system. Ideally these could be compared and matched with approaches taken in other industries. From there we could develop a more robust taxonomy of approaches and begin to assess which might be applicable in different contexts. A considerable amount of research and empirical work is needed to map the full set of strategies and interventions currently in use, who they are used by and in what context. From this point we could envisage empirical testing of different approaches and combinations of interventions, similar to those already developed for best practice and system improvement but employing a wider repertoire of approaches and, most important of all, being tested at every level of the system.

Many ideas and approaches to safety have been advanced; the very term safety has been contested and defined in numerous ways. We have a plethora of concepts and organisational ideals to guide us on the safety journey. Many of these ideas however have remained as ideas and not found a concrete expression or application. Our approach in contrast, abstract as it may seem to some, is resolutely practical in intention. The safety strategies and approaches we describe are all in use but have not been drawn together in a comprehensive architecture which attempts to embrace all healthcare settings. We have found in previous work that a unifying framework can be valuable to those managing safety at all levels of the healthcare system. We hope that our proposals and the attempt to develop an architecture of safety interventions will be useful now and productive for the future.

Open Access This chapter is distributed under the terms of the Creative Commons Attribution Noncommercial License, which permits any noncommercial use, distribution, and reproduction in any medium, provided the original author(s) and source are credited. 